\title{
Gestion positive des situations de classe : un modèle de formation en cours d'emploi pour aider les enseignants du primaire à prévenir les comportements difficiles des élèves
}

\author{
Nancy Gaudreau, Égide Royer, Claire Beaumont et Éric Frenette
}

Volume 1, mars 2012

URI : https://id.erudit.org/iderudit/1012124ar

DOI : https://doi.org/10.7202/1012124ar

Aller au sommaire du numéro

Éditeur(s)

Université Laurentienne

ISSN

1920-6275 (imprimé)

1929-8544 (numérique)

Découvrir la revue

Citer cet article

Gaudreau, N., Royer, É., Beaumont, C. \& Frenette, É. (2012). Gestion positive des situations de classe : un modèle de formation en cours d'emploi pour aider les enseignants du primaire à prévenir les comportements difficiles des élèves. Enfance en difficulté, 1, 85-115. https://doi.org/10.7202/1012124ar
Résumé de l'article

Plusieurs auteurs dénoncent depuis quelques années le manque de formation des enseignants pour gérer efficacement les comportements difficiles des élèves. Afin de soutenir la réussite des élèves qui présentent des difficultés de comportement, il s'avère essentiel de développer des modèles de formation en cours d'emploi qui répondent aux besoins des enseignants. Cet article présente le programme de formation à la gestion positive des situations de classe (GPS), spécialement conçu pour les enseignants du premier cycle du primaire à partir des données probantes de recherches dans le domaine. Il vise le développement des compétences professionnelles des enseignants en matière de gestion de la classe et des comportements difficiles. Les formules pédagogiques proposées ont pour but de soutenir le développement des croyances d'efficacité personnelle chez les enseignants afin de favoriser l'implantation de pratiques éducatives permettant de prévenir les comportements difficiles et de les gérer efficacement en classe. 
www.laurentienne.ca

\section{Gestion positive des situations de classe : un modèle de formation en cours d'emploi pour aider les enseignants du primaire à prévenir les comportements difficiles des élèves}

\section{Nancy Gaudreau \\ Université du Québec à Trois-Rivières \\ Égide Royer, Claire Beaumont et Éric Frenette \\ Université Laval}

Cet article présente un modèle de formation en cours d'emploi permettant de soutenir le développement des compétences des enseignants en matière de gestion positive des comportements difficiles en classe. Celle-ci fait référence à la gestion des comportements difficiles de certains élèves, mais également à la gestion de différentes situations de classe jugées difficiles par plusieurs enseignants (p. ex., dynamique de groupe, climat d'apprentissage difficile à instaurer, etc.). L'originalité du programme de formation à la gestion positive des situations de classe (GPS) (Gaudreau, 2008) tient au fait qu'il vise également le développement de fortes croyances d'efficacité chez les enseignants. Selon Bandura (2003), le sentiment d'efficacité personnelle de l'enseignant comprend deux sousdimensions : la croyance de l'enseignant en sa capacité à enseigner aux élèves de manière efficace (personal teaching efficacy) et la croyance que ses interventions produiront les effets escomptés chez l'élève (outcomes efficacy). Dans cet article, le sentiment d'efficacité personnelle et les croyances d'efficacité personnelle de l'enseignant sont indistinctement employés, faisant ici référence à la précédente définition. Considérant l'importance d'agir tôt auprès des élèves présentant 
des difficultés de comportement à l'école, le programme de formation GPS s'adresse particulièrement aux enseignants du premier cycle du primaire, dans la mesure où ils jouent un rôle déterminant dans la réussite éducative de ces élèves (Myers \& Pianta, 2008).

Composer avec les élèves qui présentent des comportements difficiles en classe exige beaucoup de temps de la part de l'enseignant (Gray, Miller, \& Noakes, 1996). De plus, certains comportements rendent l'enseignant plus vulnérable quant à sa capacité de mener à bien sa mission éducative au sein de la classe. Les travaux de Brouwers et Tomic (2001) ont d'ailleurs permis de mettre en lumière l'existence d'un lien entre un niveau élevé de comportements perturbateurs en classe et de faibles croyances d'efficacité personnelle en gestion de classe chez les enseignants concernés. Ainsi, plus les croyances d'efficacité de l'enseignant sont faibles, plus les comportements inappropriés en classe sont élevés. Inversement, plus ceux-ci sont fréquents, plus l'enseignant met en doute ses capacités, ce qui le rend ainsi plus vulnérable au stress et à l'épuisement professionnel. À cet effet, Poulou (2005) précise : « Il est évident que les enseignants jouent un rôle déterminant auprès des élèves qui présentent des troubles de comportement. L'inverse est aussi vrai, ces élèves représentent le plus grand défi des enseignants sur le plan personnel et professionnel » (p. 138). C'est pourquoi les formules pédagogiques proposées dans le programme de formation à la gestion positive des situations de classe (GPS) présenté dans cet article visent le développement des croyances d'efficacité chez les enseignants afin de favoriser l'implantation de pratiques éducatives adéquates pour prévenir les comportements difficiles et agir efficacement pour y faire face dans la salle de classe.

\section{Enseigner aux élèves présentant des comportements difficiles}

La gestion efficace des comportements difficiles en classe exige avant tout la mise en place de mesures permettant de prévenir l'indiscipline. Puisque le concept de gestion de classe est au cœur de la formation des enseignants et qu'il peut être interprété de différentes manières, la définition proposée par Evertson et Weinstein (2006) a été retenue dans la mesure où elle apporte un éclairage intéressant. Elle stipule que la gestion de classe est constituée d'actions posées par l'enseignant qui permettent de créer un environnement qui soutient et facilite l'apprentissage scolaire et social (p. 4, traduction libre). Ainsi, la 
gestion de classe ne fait pas uniquement référence à la discipline, mais aussi à la création d'un environnement propice au développement intellectuel, social et moral des élèves. Pour faire œuvre d'éducation auprès des élèves présentant des comportements difficiles, l'enseignant doit d'abord maîtriser les ficelles d'une bonne gestion de classe (mesures universelles), puis connaître la meilleure façon de composer efficacement avec des élèves ayant des difficultés de comportement à l'aide de pratiques exemplaires. En milieu scolaire, les problèmes de comportement sont définis en fonction de leur fréquence, de leur durée, de leur intensité et de leur constance (MELS ${ }^{1}$, sous presse). Ces critères permettent entre autres de distinguer les élèves qui présentent des difficultés de comportement de ceux qui présentent des troubles du comportement. Les difficultés comportementales se traduisent souvent par des comportements de désobéissance répétée liés à des facteurs environnementaux, tandis que les troubles du comportement sont des problèmes d'adaptation plus importants, qui se manifestent avec constance dans plusieurs sphères de la vie de l'élève.

Plusieurs études se sont penchées sur les effets des attitudes et des pratiques éducatives des enseignants sur les comportements des élèves. Hamre et Pianta (2001) ont révélé que les élèves qui présentent des difficultés comportementales à la maternelle présentent moins de problèmes plus tard à l'école si leur enseignant est sensible à leurs besoins et leur prodigue des rétroactions positives fréquentes au début de leur scolarisation. Helm (2007) ajoute que même si les élèves ne bénéficient pas du soutien de leurs parents, ils peuvent bien réussir à l'école si leurs enseignants s'engagent à s'investir auprès d'eux. Pour ce faire, les enseignants doivent être en mesure de faire preuve de sensibilité, d'éthique et de positivisme.

Pour sa part, Ellis (1997) rappelle de manière originale l'importance de la relation maître-élève dans la gestion des comportements en classe tel que le rapporte ce témoignage de Terry, 12 ans, invité à commenter sa perception d'une bonne relation maître-élève :

Gardez votre porte de classe ouverte quand les élèves arrivent. Accueillez-les à la porte et parlez avec eux. Intéressez-vous à eux, ils vous comprendront et ils cesseront de se moquer de vous après la classe. Prenez le temps d'apprendre à les connaître, demandez-leur ce qu'ils font de leurs soirées ou sur l'heure du dîner. (Ellis, 1997, p. 19, traduction libre) 
Les élèves attendent de leurs enseignants qu'ils mettent en place des routines, qu'ils adaptent leur enseignement en fonction de leurs capacités, qu'ils les encouragent et les félicitent (Ellis, 1997). Dans le même sens, les résultats des études menées par Buyse, Verschueren, Doumen, Van Damme et Maes (2008) soulignent l'importance d'établir une relation positive entre l'enseignant et les élèves présentant des comportements difficiles en classe. Toutefois, ils précisent que l'intervention ne doit pas être dirigée uniquement vers l'élève, comme c'est souvent le cas. Selon Hamre et Pianta (2005), davantage d'efforts doivent être concentrés sur le climat de la classe, puisqu'il exerce une influence importante sur le développement des problèmes de comportement à l'école. Conséquemment, l'enseignant doit, de toute évidence, établir un lien de confiance avec les élèves tout en ayant recours à des pratiques efficaces de gestion de la classe.

\section{La préparation des enseignants}

Plusieurs recherches ont mis en évidence la présence d'une relation positive entre les comportements difficiles des élèves, le stress vécu par les enseignants (Alvarez, 2007; Kovess-Masféty, Rios-Seidel, \& SevillaDedieu, 2007; Wisniewski \& Gargiulo, 1997), ainsi que l'épuisement professionnel et l'abandon de la profession (Brouwers \& Tomic, 2000; Burke, Greenglass, \& Shwarzer, 1996; Gersten, Keating, Yovanoff, \& Harniss, 2001; Hastings \& Bham, 2003).

Certaines études démontrent que le bien-être et la satisfaction au travail des enseignants sont également associés à leur capacité à gérer efficacement les situations difficiles qui se présentent en classe (Perkins \& Leadbetter, 2002). Toutefois, nombre d'écrits, tant scientifiques que professionnels, mentionnent que la préparation des enseignants en matière d'intervention auprès des élèves présentant des difficultés de comportement est insuffisante (Begeny et Martens, 2006; Conseil des ministres de l'Éducation, 2002; Conseil supérieur de l'éducation (CSE), 2001; Couture, 2005; Jeffrey \& Sun, 2006; Merrett \& Wheldall, 1993; Ndoreraho \& Martineau, 2006; Rosenberg, Sindelar, \& Hardman, 2004; Royer, 2006).

Selon les enseignants interrogés au cours de l'étude de Merrett et Wheldall (1993), les habiletés de gestion de classe sont d'une importance majeure dans leur travail. Cependant, près du tiers des enseignants parmi les répondants affirment ne pas être suffisamment formés dans ce domaine. Dans le même sens, les travaux de Jeffrey et Sun (2006) 
révèlent que seulement un enseignant sur cinq possède, lors de son entrée sur le marché du travail, une connaissance suffisante des différentes stratégies d'intervention adaptées aux élèves présentant des difficultés de comportement. La présence d'un fossé important entre les connaissances et la pratique dans le domaine de la discipline et de l'intervention comportementale auprès des élèves rend les enseignants très vulnérables au stress vécu lors de la gestion des comportements difficiles en classe. Selon les travaux de Dembo et Gibson (1985), certains individus peuvent développer un sentiment d'inadéquation lorsqu'ils réalisent qu'ils ne possèdent pas les connaissances ou les habiletés nécessaires pour composer avec les situations auxquelles ils ont à faire face. Ce sentiment d'inadéquation ou d'incompétence incite ainsi les enseignants à adopter des stratégies d'évitement des situations perçues comme difficiles à gérer. D'ailleurs, comme le mentionnent Mayer (2001) et Royer (2006), les enseignants ont trop souvent recours à des méthodes punitives pour gérer les comportements perturbateurs en classe. Cette pratique a pour effet de nuire à la relation maîtreélève, en favorisant la création d'un cercle vicieux de coercition menant au développement de problèmes de comportement plus importants (Patterson, Reid, \& Dishion, 1992; Walker, Ramsey, \& Gresham, 2004). Pour Skiba et Peterson (2000), certaines écoles se retrouveraient aussi avec des ressources insuffisantes ou mal préparées pour faire face aux problèmes liés à l'indiscipline et à la violence. La formation continue des enseignants devient donc un moyen privilégié pour développer leur sentiment de compétence en gestion des comportements difficiles en classe. Cela a aussi pour effet de favoriser la mise en œuvre de pratiques d'intervention éducatives non aversives.

\section{Formation continue et gestion des comportements difficiles en classe}

La formation en cours d'emploi est perçue comme un excellent moyen de développement professionnel (McEvoy, McDonnell, \& Dearden, 1991). Très souvent, la formation continue des enseignants prend la forme du modèle Train and Hope de Milne (1986) voulant que l'on offre une formation en espérant qu'elle produise les effets escomptés. Les recherches portant sur le développement professionnel des enseignants sont, la plupart du temps, menées en contexte universitaire ou dans des conditions qui répondent d'abord aux exigences de la recherche. Rares sont celles qui ont été réalisées en milieu scolaire auprès d'enseignants en exercice dans leur contexte naturel (Shernoff \& Kratochwill, 2007). 
Elles sont aussi peu nombreuses à viser l'évaluation de modèles de formation destinés aux enseignants sur le marché du travail afin d'en évaluer les effets (Garet, Porter, Desimone, Birman, \& Yoon, 2001). Bref, bien que plusieurs chercheurs, dont Murik, Shaddock, Spinks, Zilber et Curry (2005), recommandent fortement la formation en cours d'emploi, la Coalition for Evidence-Based Policy ${ }^{1}$ (2002) stipule que peu de recherches visent à en évaluer l'efficacité.

\section{Les effets de la formation sur la qualité de l'intervention en classe}

Il a été démontré que le développement professionnel des enseignants en gestion de la classe et des comportements représente un facteur clé dans la trajectoire comportementale des élèves du primaire (Lindauer, Petrie, Garth, \& Vickers, 1998) et dans la réussite de leur inclusion à l'école (Dickens-Smith, 1995). De fait, plusieurs études révèlent que les enseignants mieux formés sont plus aptes à répondre aux besoins de leurs élèves en créant un environnement de classe positif pour l'ensemble d'entre eux (Allen \& Blackston, 2003; Evertson, 1989; Lewis, 2001; Melby, 1995; Sawka, McCurdy, \& Mannella, 2002; Swinson \& Cording, 2002; Veenman, Lem, \& Roelofs, 1989). Pour Alvarez (2007), des enseignants mieux formés sont également plus efficaces dans leur réponse aux comportements agressifs des élèves et réussissent davantage à enseigner des comportements de remplacement aux élèves présentant des difficultés.

\section{Les effets de la formation sur les croyances d'efficacité des enseignants}

À l'aide du développement des compétences professionnelles des enseignants, on augmente les probabilités que ceux-ci vivent des expériences de succès, favorisant ainsi la croissance des croyances d'efficacité personnelle (Bandura, 2003). D'ailleurs, plusieurs chercheurs ont observé des effets particulièrement positifs sur le sentiment d'efficacité personnelle des enseignants lorsque les activités de formation visent à les mobiliser dans l'application de stratégies pédagogiques enseignées dans leur pratique professionnelle (Fritz, Miller-Heyl, Kreutzer, \& MacPhee, 1995; Lewis, 2001; Rimm-Kaufman \& Sawyer, 2004; Ross, 1994; Stein \& Wang, 1988). Pour Robardey, Allard et Brown (1994), de même que pour Ross (1994), les programmes ayant prévu des moments d'interactions entre les participants obtiennent également de meilleurs résultats. Ces auteurs rejoignent la thèse 
de Bandura (2003) voulant que cette modalité d'animation crée des opportunités où les enseignants peuvent témoigner de leur réussite, ce qui constitue un excellent moyen persuasif pour inciter leurs pairs à expérimenter de nouvelles stratégies d'enseignement.

\section{Les ingrédients essentiels à une formation en cours d'emploi}

Certaines recherches portant sur le processus de changement de pratiques chez les enseignants révèlent qu'offrir une formation axée uniquement sur le développement de connaissances et d'habiletés est inefficace (Ohlhausen, Meyerson, \& Sexton, 1992; Stein \& Wang, 1988). Pour Bissonnette et Richard (2010) et Fritz et al. (1995), des programmes capables de répondre aux besoins exprimés par les enseignants et qui prévoient des mesures de soutien efficaces sont nécessaires. D'autres chercheurs mentionnent que les approches traditionnelles de développement professionnel comme les conférences et les ateliers de formation d'une journée suscitent l'intérêt des enseignants, mais ne permettent pas de provoquer de changements de pratiques durables chez ceux-ci (Corcoran, Shields, \& Zucker, 1998; Hustler et al., 2003; Shields, Marsh, \& Adelman, 1998; Weiss, Montgomery, Ridgway, \& Bond, 1998). Par ailleurs, Levin et Rock (2003) mentionnent que les activités de développement professionnel à plus long terme permettent, pour leur part, d'intégrer une pratique réflexive nécessaire à l'analyse critique des pratiques, élément préalable au changement. C'est pourquoi plusieurs chercheurs insistent sur l'importance de prévoir des séances de formation en cours d'emploi qui s'échelonnent dans le temps et qui prévoient des mesures de soutien et d'accompagnement pour les enseignants qui en ressentent le besoin (Bissonnette \& Richard, 2010; Hoffman, Hutchinson, \& Reiss, 2009; Jones \& ChronisTuscano, 2008; Joyce \& Showers, 2002; Sheilds, Marsh, \& Adelman, 1998; Shernoff \& Kratochwill, 2007; Weiss et al., 1998). Selon Kariuki (2009), un développement professionnel de haut niveau se réalise sur une période de temps suffisamment longue (voire de plusieurs années) pour provoquer un changement de pratiques chez l'enseignant, ce qui est difficile à faire lors d'une activité de formation très courte (p. ex., une activité de formation d'une journée, sans suivi).

Les activités de formation permettent, certes, de contribuer positivement au développement des croyances d'efficacité des enseignants. Néanmoins, l'inverse est aussi vrai. Par l'influence qu'elles exercent sur l'implication des enseignants lors des activités de perfectionnement, 
les croyances d'efficacité personnelle des enseignants influencent les effets de la formation reçue. Comme le mentionnent Carleton, Fitch et Krockover (2008), les enseignants qui présentent un fort sentiment d'efficacité personnelle sont plus enclins à expérimenter de nouvelles méthodes pédagogiques et technologiques et démontrent plus d'enthousiasme envers l'enseignement. Par conséquent, plus l'enseignant possède de fortes croyances d'efficacité personnelle, plus les activités de formation auxquelles il participe produisent les effets escomptés. Ainsi, les croyances d'efficacité de l'enseignant exercent une influence sur son implication dans les activités relatives à son développement professionnel (Carleton et al., 2008). Baker, Kupersmidt, Voegler-Lee, Arnold et Willoughby (2010) ajoutent que la qualité de la participation aux activités de formation est également influencée par les croyances relatives à la présence de soutien au sein de l'équipe scolaire, à la satisfaction au travail et à l'engagement dans celui-ci.

En résumé, la littérature consultée fait ressortir le fait que la formation en cours d'emploi sera plus efficace si elle satisfait les critères de réussite mentionnés par les scientifiques qui ont étudié le développement du sentiment d'efficacité personnelle et la formation en cours d'emploi. Ces critères se définissent ainsi :

1) la formation doit répondre aux besoins exprimés par les enseignants;

2) elle doit se dérouler sur une période de temps suffisamment longue (plusieurs mois, voire des années);

3) elle doit offrir des mesures de soutien et d'accompagnement individualisées (rencontres, appels téléphoniques, visites dans les classes);

4) elle doit proposer des activités qui incitent les enseignants à expérimenter de nouvelles pratiques;

5) elle doit favoriser le soutien par les pairs.

\section{Modèle de formation à la gestion des comportements difficiles en classe}

Le programme de formation GPS (Gaudreau, 2008) vise à développer les compétences des enseignants en matière de gestion des comportements difficiles en classe. Inspiré par la théorie sociocognitive de l'autoefficacité (sentiment d'efficacité personnelle) de Bandura (1977, 1986, 1991, 2003), par les travaux de Garet et al. (2001), Ross (1994), Ross et Bruce (2007), Wheldall et Merrett (1992) et Siegel et Jausovec (1994), le 
programme GPS soutient que certains principes de base sont nécessaires pour assurer la qualité d'une formation en cours d'emploi susceptible de provoquer des changements de pratiques en regard de la gestion des comportements difficiles en classe chez les enseignants en exercice :

1) Le formateur et les participants doivent adhérer à la philosophie du programme voulant que l'expérimentation, les échanges entre collègues et la réflexion sur sa pratique facilitent la mise en œuvre de nouvelles pratiques éducatives en classe;

2) Les participants doivent reconnaître l'influence du climat relationnel de la classe sur la conduite des élèves;

3) Les participants doivent reconnaître l'influence réciproque exercée par les comportements de l'élève et de l'enseignant;

4) Les participants doivent s'impliquer activement dans les activités de développement professionnel proposées et le formateur doit leur permettre de réfléchir sur des situations problématiques réelles;

5) La formation doit répondre aux besoins exprimés par les participants et le formateur doit procéder à une évaluation continue des activités de formation afin d'effectuer les ajustements nécessaires;

6) Les participants doivent être amenés à percevoir les situations difficiles en classe comme étant de bonnes opportunités d'apprentissage;

7) Le formateur doit reconnaître la complexité des compétences professionnelles nécessaires chez les enseignants qui œuvrent auprès des élèves présentant des comportements difficiles;

8) Les participants doivent apprendre à délaisser le modèle d'intervention intuitive au profit de méthodes d'intervention s'inspirant de l'avancement des connaissances dans le domaine. Ils doivent aussi concevoir les élèves et leur développement comportemental comme étant uniques afin d'éviter de reproduire les mêmes interventions pour les mêmes problématiques comportementales.

Lorsqu'elles sont respectées, ces conditions viennent bonifier les chances que les activités de formation continue produisent les effets escomptés chez les enseignants participants. Dans le cas contraire, la formation risque d'être moins efficace à susciter la mise en œuvre de pratiques d'intervention renouvelées au sein de ce groupe. 


\section{Soutenir le développement des croyances d'efficacité des enseignants}

Pour Gordon (2001), il existe une relation positive entre le sentiment d'efficacité personnelle de l'enseignant et sa capacité à gérer efficacement les comportements difficiles des élèves. Il est donc primordial de prévoir des moyens susceptibles d'accroître le sentiment d'efficacité des enseignants pendant leur développement professionnel (Baker, 2005). À cet effet, Bandura (2003), dans sa théorie sociocognitive de l'auto-efficacité, a identifié quatre sources spécifiques qui contribuent à façonner le sentiment d'efficacité personnelle des enseignants: l'expérience de maîtrise, l'expérience vicariante, la persuasion verbale et les états émotionnels et physiologiques.

\section{L'expérience de maîtrise}

Considérée comme étant la source la plus puissante d'efficacité, l'expérience de maîtrise fait référence au fait de vivre une expérience de succès dans un contexte donné. Chaque expérience de maîtrise contribue à l'augmentation des croyances d'efficacité dans le même champ d'activités. À l'inverse, les expériences antérieures d'échec affectent de manière négative le sentiment d'efficacité personnelle de la personne. Dans le cadre des activités de formation du programme GPS, afin de favoriser les opportunités d'expériences de maitrise, certains moyens de développement professionnel jugés efficaces par Ross et Bruce (2007) sont privilégiés : présentation d'exemples tirés de la pratique; activités de modelage de stratégies efficaces; opportunités de réflexion sur sa pratique; mise en pratique en contexte réel et rétroaction. Ainsi, les enseignants sont invités à planifier des objectifs précis et réalistes à court terme. Par le fait même, l'auto-évaluation est facilitée.

\section{L'expérience vicariante}

Seconde source d'efficacité personnelle, l'expérience vicariante s'actualise lorsqu'un pair jugé équivalent (de même statut ou ayant des aptitudes similaires) vit une réussite. Au cours de cette expérience, l'individu développe la confiance en sa propre capacité de réussir à son tour là où son collègue a vécu du succès. Lobservation des pairs permet aussi aux individus d'apprendre. D'après Bandura (1986), l'apprentissage vicariant est un apprentissage socioconstructif par observation. Ainsi, l'observation d'une personne performante dans une activité 
donnée peut renforcer le sentiment d'efficacité personnelle, notamment dans le cas où l'observateur n'aurait que peu d'expérience dans ladite activité. À l'inverse, la confrontation à l'échec de ses pairs risque de l'affaiblir. Puisque les croyances d'efficacité personnelle de l'enseignant se développent en observant ses pairs réussir auprès de leurs élèves, les modalités d'animation des rencontres de formation du programme GPS favorisent les échanges d'expériences entre participants. Pour ce faire, chacune des rencontres de formation débute par une activité de partage favorisant le témoignage au sujet d'expériences de réussite communément appelées "les bons coups ", tel que proposé par Ross (1994). Les activités de résolution de problèmes donnent également lieu à des échanges constructifs entre collègues, constituant ainsi de nouvelles expériences vicariantes.

\section{La persuasion verbale}

Selon Bandura (2003), le fait d'être entouré et soutenu par des personnes qui nous encouragent à poursuivre nos objectifs et à surmonter les difficultés a un effet positif sur les croyances d'efficacité de la personne. Les sujets que l'on persuade de posséder les capacités de maîtriser certaines activités ont plus de chances de produire un effort supplémentaire et de le maintenir que ceux qui doutent d'eux-mêmes et qui évoquent des lacunes personnelles quand surviennent les difficultés (Poulou, 2007). Particulièrement importante dans le développement des croyances d'efficacité des jeunes enseignants (Ross, 1994; Ross \& Bruce, 2007), la persuasion verbale s'actualise, dans le cadre des activités de formation du programme GPS, par la présence de rétroactions positives et fréquentes entre les pairs et entre le formateur et les participants tel que recommandé par Baker (2005). Lors des ateliers de formation GPS, le formateur incite les enseignants à participer activement aux activités proposées et à recourir à de nouvelles pratiques éducatives auprès des élèves présentant des comportements difficiles en classe. Il leur fait part de sa confiance en leurs capacités et leur rappelle qu'il demeure disponible pour les soutenir dans leur démarche.

Toutefois, la persuasion verbale du formateur ne sera efficace que si celui-ci est jugé compétent et expérimenté aux yeux des participants :

L'impact des opinions persuasives sur les croyances d'efficacité dépend de la confiance que le destinataire a de la personne qui les exprime. La confiance est médiatisée par la crédibilité perçue 
et par l'expertise de l'individu cherchant à persuader le sujet. Les gens ont tendance à croire l'évaluation de leurs capacités par ceux qui sont eux-mêmes doués dans l'activité ou qui possèdent un riche fond de connaissances acquises en observant et en comparant de nombreux candidats différents ainsi que leurs réalisations ultérieures (Crundall \& Foddy, 1981; Wenster \& Sobieszek, 1974, cités dans Bandura, 2003 p. 161).

L'étude de Parent et al. (1997) auprès d'enseignants en exercice soutient également cette thèse. Les enseignants interrogés sur leurs besoins en formation continue désirent recourir à des personnes renommées capables de leur offrir une formation sur mesure, partant de leur vécu. Ils disent aussi accorder plus de crédibilité à la personne qui anime la session de formation qu'au contenu de celle-ci. Le pouvoir d'influence de la personne qui prend en charge l'animation de la formation des enseignants est réel. Il faut donc s'assurer de sa crédibilité pour obtenir des résultats optimaux (Bissonnette \& Richard, 2010; Skaalvik \& Skaalvik, 2007).

\section{Létat psychologique et physiologique}

Dans la mesure où l'individu peut réguler ce qu'il pense, il peut influencer sa manière de se sentir et de se comporter. Lintervention auprès d'élèves présentant des difficultés de comportement est souvent source de stress (Hastings, 2005). Les enseignants qui éprouvent de la difficulté à contrôler leur pensée ont plutôt tendance à réagir passivement aux événements, victimes de la situation, au lieu d'être efficaces et en contrôle de leurs actions. Ainsi, les émotions négatives, le stress et la nervosité communiquent de l'information négative à propos des compétences (Bandura, 2003; Ross \& Bruce, 2007). La régulation des états émotionnels par l'efficacité du contrôle de la pensée joue alors un rôle important. L'efficacité du programme de formation GPS est tributaire de sa capacité à accompagner les enseignants vers la recherche d'un meilleur contrôle des pensées et des actions en contexte d'intervention lors de situations difficiles en classe. La planification de scénarios efficaces d'intervention, les activités régulières de réflexion sur sa pratique et la mise en place de stratégies personnelles pour mieux gérer son stress sont des exemples de moyens proposés dans le cadre du programme de formation GPS. 


\section{Compétences visées par le programme de formation GPS}

Puisque le programme de formation GPS s'inscrit dans un contexte de formation continue des enseignants, il s'impose de cibler les compétences professionnelles visées par les contenus enseignés et les activités d'intégration réalisées. La notion de compétence fait référence à la capacité de savoir-agir, qui exige la mise en œuvre du savoir (les connaissances), du savoir-faire (les habiletés) et du savoir-être (les attitudes) en contexte réel d'action (MEQ, 2001, p. 50). Pour réussir à intervenir efficacement auprès des élèves présentant des comportements difficiles, les enseignants doivent ainsi développer des compétences variées. Par conséquent, la mise à jour des connaissances en la matière (à l'aide de lectures et d'ateliers de formation), le développement d'habiletés d'intervention (à l'aide d'expérimentations en classe et de témoignages vicariants) et le développement d'attitudes d'ouverture (à l'aide de témoignages vicariants, de persuasion verbale et d'expériences de maîtrise) s'avèrent essentiels. À partir du cadre de référence sur la formation à l'enseignement du MEQ (2001), les compétences suivantes ont été ciblées dans le cadre du programme GPS :

1) Planifier, organiser et superviser le mode de fonctionnement $\mathrm{du}$ groupe-classe en vue de favoriser l'apprentissage et la socialisation des élèves;

2) Adapter ses interventions aux besoins et aux caractéristiques des élèves présentant des difficultés d'apprentissage, d'adaptation ou un handicap;

3) Coopérer avec l'équipe-école, les parents, les différents partenaires sociaux et les élèves en vue de l'atteinte des objectifs éducatifs de l'école;

4) Travailler de concert avec les membres de l'équipe pédagogique à la réalisation des tâches permettant le développement et l'évaluation des compétences visées dans le programme de formation, et ce, en fonction des élèves concernés;

5) S'engager dans une démarche individuelle et collective de développement professionnel;

6) Agir de façon éthique et responsable dans l'exercice de ses fonctions.

La sélection de ces compétences a été effectuée en tenant compte de leurs composantes respectives et des liens qu'elles entretiennent avec les contenus de formation. 


\section{Contenus des formations offertes dans le cadre du programme GPS}

La lecture d'ouvrages portant sur la gestion des comportements en classe a permis de dégager les conditions essentielles à l'intervention efficace auprès des élèves présentant des comportements difficiles.

Parmi les habiletés les plus importantes à développer chez les enseignants, la capacité de donner des directives claires joue un rôle central (Bertsch, Houlihan, Lenz, \& Patte, 2009; Canter \& Canter, 2001). Celle-ci facilite, entre autres, l'établissement d'un climat propice à l'apprentissage (Matheson \& Shriver, 2005). Badia Martin (2006) ajoute que la capacité à établir des routines, à donner des rétroactions positives et à demander le soutien des parents et de ses collègues est essentielle pour intervenir efficacement auprès des élèves présentant des comportements difficiles à l'école. Selon Woolfolk (2004, p. 21), cela se traduit par le respect de tous les élèves et de leurs parents en toute circonstance, la reconnaissance du potentiel de chacun et la capacité de faire preuve de spontanéité et de créativité afin de reconnaître les moments propices aux apprentissages.

À la lumière des travaux d'Archambault et Chouinard (2005, 2009), Beaman et Wheldall (2000), Doyle (2006), Evertson (1989), Evertson et Emmer (2009), Evertson et Weinstein (2006) et Jones et Jones (2007) portant sur les conditions préalables à une gestion de classe efficace, ainsi que de ceux de Bertsch et al. (2009), Boynton et Boynton (2009), Canter et Canter (2001), Jolivette et Steed (2010), Kauffman, Mostert, Trent et Pullen (2006), Kehle, Bray, Theodore, Jenson et Clark (2000) et Massé, Desbiens et Lanaris (2006) portant sur la gestion de comportements difficiles en milieu scolaire, voici une synthèse des conditions essentielles à une intervention efficace auprès des élèves présentant des comportements difficiles :

1) Établir et maintenir l'ordre et la discipline dans la classe en annonçant des attentes claires et en élaborant un système de règles et de procédures cohérent où les conséquences aux manquements sont connues par les élèves;

2) Enseigner aux élèves à bien se comporter et favoriser leur participation aux décisions de la classe;

3) Établir des routines et donner des rétroactions positives fréquentes;

4) Obtenir le soutien des parents, des collègues et de la direction; 
5) Anticiper les problèmes et ramener rapidement les élèves à l'ordre;

6) Observer et analyser les effets des interventions afin d'ajuster ses pratiques de gestion de classe;

7) Intervenir de manière différenciée, rapide et efficace afin de diminuer la fréquence des comportements difficiles souvent maintenue, voire augmentée, par l'attention inadéquate qu'ils obtiennent de la part de l'enseignant;

8) Respecter tous les élèves et leurs parents en toute circonstance.

Il va sans dire que la présence d'une relation maître-élève positive est essentielle. Il s'agit là d'un préalable à toute intervention visant à accompagner l'élève ayant des difficultés de comportement. Le programme de formation GPS a été élaboré en accordant une attention particulière au développement des compétences des enseignants afin qu'ils soient en mesure d'adopter des pratiques éducatives respectueuses des conditions essentielles à une intervention efficace auprès des élèves présentant des comportements difficiles. Le choix des contenus de formation a été effectué en tenant compte de l'avancement de la recherche en gestion de la classe et en gestion des comportements tel que présenté précédemment.

L'étude de Bullock, Ellis et Wilson (1994) a permis de répertorier 201 éléments portant sur des connaissances, des habiletés et des attitudes à développer chez les enseignants qui œuvrent auprès d'élèves présentant des troubles du comportement. Les 102 enseignants consultés lors de cette étude ont classé chacun des éléments répertoriés selon leur importance, leur fréquence d'utilisation et leur niveau de compétence à les mettre en œuvre. Les résultats de cette étude permettent de dégager certains éléments de contenus jugés essentiels pour la formation des enseignants à l'intervention auprès des jeunes présentant des comportements difficiles en classe. De plus, selon le Conseil supérieur de l'éducation (2001), la formation continue des enseignants doit porter sur la gestion de la classe, l'intervention en situation de crise, l'intervention auprès d'élèves présentant des troubles de la conduite et du comportement et la gestion des problèmes disciplinaires susceptibles de survenir au sein de groupes d'enfants. Les choix de contenus du programme de formation GPS ont été réalisés selon les recommandations issues des travaux de Bullock et al. (1994) et du CSE (2001). Ils sont présentés dans le Tableau 1. 


\section{Tableau 1 - Contenus de formation du programme GPS}

\begin{tabular}{|c|c|}
\hline Ateliers & Sujets traités \\
\hline 1 & $\begin{array}{l}\text { - La gestion de la classe : mieux vaut prévenir que } \\
\text { punir } \\
\text { - Les mesures préventives en classe }\end{array}$ \\
\hline 2 & $\begin{array}{l}\text { L'observation et le dépistage des difficultés de } \\
\text { comportement en classe } \\
\text { - Les caractéristiques de l'élève en difficulté de } \\
\text { comportement à l'école }\end{array}$ \\
\hline 3 & $\begin{array}{l}\text { - L'analyse fonctionnelle du comportement } \\
\text { - L'intervention individualisée }\end{array}$ \\
\hline 4 & $\begin{array}{l}\text { - Les techniques d'intervention non-aversives } \\
\text { - Les pratiques exemplaires }\end{array}$ \\
\hline 5 & - Lintervention auprès de l'élève présentant un TDAH \\
\hline 6 & $\begin{array}{l}\text { - Les comportements agressifs chez les jeunes élèves } \\
\text { - Lintervention face aux comportements d'opposition } \\
\text { - Lintervention en situation de crise }\end{array}$ \\
\hline 7 & $\begin{array}{l}\text { - La gestion du stress } \\
\text { - La collaboration et la communication avec les } \\
\text { parents }\end{array}$ \\
\hline 8 & - Le développement des habiletés sociales \\
\hline
\end{tabular}

\section{Modalités d'application du programme de formation GPS auprès d'enseignants en cours d'emploi}

Le programme de formation GPS prévoit huit rencontres mensuelles d'une durée de trois heures chacune sur une période d'une année scolaire. Cela permet d'augmenter la portée du programme auprès des participants (Corcoran, 1995; Darling-Hammond, 1995; Lieberman, 1996; Little, 1993). Le programme prévoit aussi la mise en place de dispositifs favorisant l'établissement de pratiques collaboratives (en personne ou de manière virtuelle) entre les participants ainsi qu'avec le formateur, tel que le recommandent plusieurs chercheurs (Bissonnette \& Richard, 2010; Boyle, Lamprianou, \& Boyle, 2005). Par exemple, en accédant au portail des enseignants de la commission scolaire, les participants peuvent rejoindre la "communauté GPS" afin d'échanger des documents (dépôts et téléchargements) avec les autres membres (autres enseignants participants et formateur). Ils peuvent aussi 
communiquer par courrier électronique, à l'aide du forum de discussion ou encore par clavardage.

Afin de soutenir les enseignants dans l'expérimentation de nouvelles pratiques éducatives en classe, une banque d'outils est proposée aux participants (les outils GPS). Spécialement conçus pour répondre aux besoins des enseignants et des élèves du premier cycle du primaire, ces outils visent à faciliter le transfert des connaissances dans la pratique. Ils prennent la forme de modèles d'affiches pour l'encadrement (règles, responsabilités, horaire), de stratégies de résolution de problèmes et de développement d'habiletés sociales, de formulaires d'observation, de planification de l'intervention et de fiches de réflexion sur sa pratique. Des exemples d'activités à animer en classe pour stimuler la motivation des élèves à bien se comporter sont également proposés. Aussi, afin de soutenir la réflexion et l'apprentissage, chacun des ateliers de formation s'accompagne de lectures. Pour ce faire, un recueil de textes est offert à tous les participants lors de la première rencontre de formation. Ce recueil est composé d'extraits de publications ministérielles, d'articles professionnels et scientifiques et de chapitres de livres portant sur le sujet. La figure 1 présente les caractéristiques du déroulement des activités de formation GPS dans le temps.

\section{Figure 1 - Déroulement des activités de formation GPS}

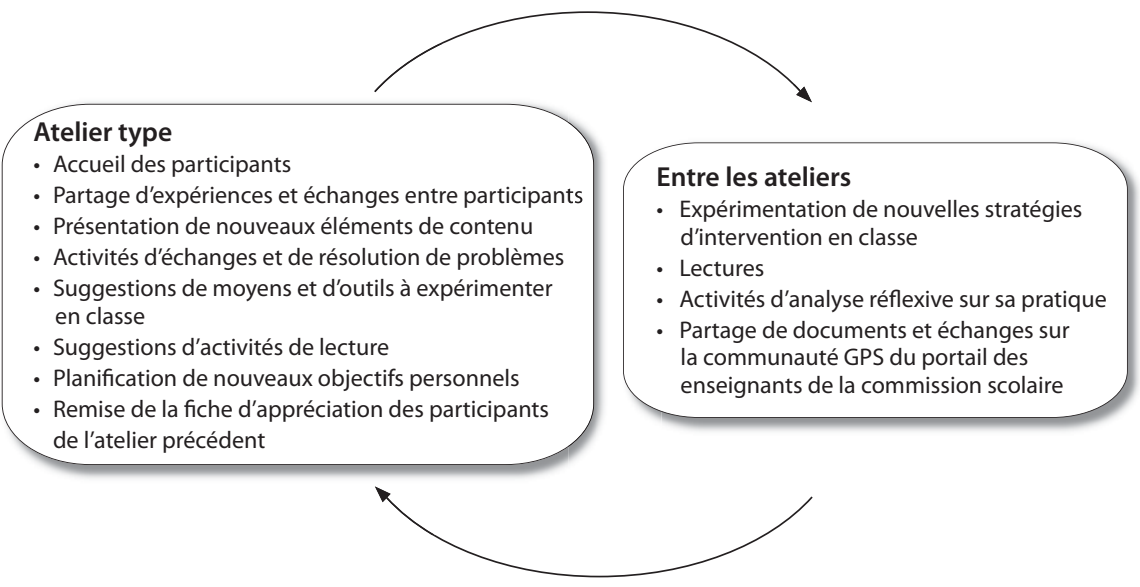

\section{Effets attendus auprès des enseignants ayant participé au programme GPS}

Développé selon les recommandations issues d'une recension des écrits (portant sur le développement professionnel des enseignants, 
le développement de leurs croyances d'efficacité et la gestion des comportements en classe), le programme de formation GPS constitue un moyen prometteur pour soutenir la réussite éducative des élèves, tel qu'illustré par la figure 2 .

\section{Figure 2 - Effets escomptés de la participation au programme de formation GPS}

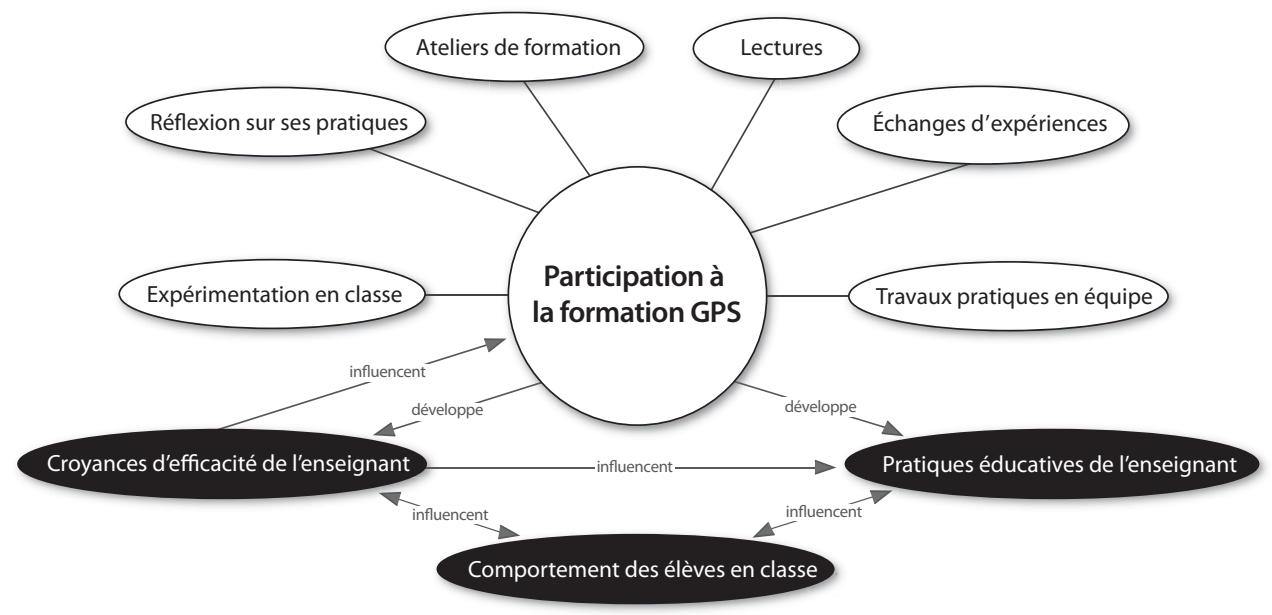

Les résultats des recherches antérieures permettent de croire qu'en animant des activités de formation qui favorisent la mise en place de pratiques collaboratives entre enseignants, en incitant ceux-ci à expérimenter de nouvelles pratiques éducatives en classe et en leur proposant de réfléchir sur leurs pratiques, le programme de formation GPS soutient la mise en œuvre des différentes sources d'efficacité présentées précédemment. Aussi, en développant de meilleures croyances d'efficacité personnelle par l'actualisation de pratiques éducatives qui permettent de gérer efficacement les comportements difficiles, les enseignants peuvent influencer le comportement de leurs élèves. Le comportement des élèves influence à son tour les pratiques éducatives de l'enseignant et ses croyances d'efficacité personnelle, mais le processus par lequel ces changements opèrent exige du temps. C'est pourquoi le programme de formation GPS s'échelonne sur plusieurs mois. Ainsi, lors de chacune des rencontres mensuelles, il devient plus facile de provoquer des moments d'échanges vicariants qui soutiennent le développement des croyances d'efficacité personnelle des enseignants. Par exemple, lors des activités de pratique réflexive, 
l'enseignant identifie peu à peu les situations jugées les plus difficiles et les solutions envisageables afin d'intervenir plus efficacement en prenant soin de planifier des scénarios d'intervention qui favorisent un meilleur contrôle de ses états physiologiques et émotionnels.

\section{Le processus de changement de pratique chez les enseignants en exercice}

À la lumière de leur recension des écrits en éducation et en psychologie, Stein et Wang (1988) ont identifié certains facteurs qui favorisent l'appropriation de nouvelles pratiques pédagogiques chez les enseignants en formation. Leur modèle conceptuel attribue un rôle central à deux composantes dans le processus de changement : les croyances d'efficacité de l'enseignant dans l'implantation de nouvelles approches pédagogiques et la valeur qu'il accorde au programme. Toutes deux jouent un rôle important dans le succès du changement de pratiques et dans la motivation de l'enseignant à persévérer dans l'acquisition de nouvelles connaissances. Selon ces chercheurs, l'auto-évaluation de l'enseignant, basée sur les résultats des élèves et les rétroactions des différents acteurs du milieu de l'éducation, influence à son tour son sentiment d'efficacité personnelle. La perception de l'efficacité du programme, du rôle joué par l'école ainsi que de ses objectifs professionnels et personnels influence, pour sa part, la valeur perçue du programme et des pratiques que l'enseignant propose.

Les travaux de Ross et Bruce (2007), basés sur le modèle théorique de l'auto-efficacité (sentiment d'efficacité personnelle) de Bandura (2003), ont aussi permis de dresser un modèle intéressant permettant d'illustrer le processus de changement de pratiques chez l'enseignant en formation où l'auto-évaluation joue un rôle central. Selon le modèle de Ross et Bruce (2007), l'enseignant se base sur l'observation des réalisations de ses élèves pour porter un jugement sur l'atteinte des objectifs pédagogiques fixés. Ce jugement influence ainsi son degré de satisfaction personnelle (réaction). Ce modèle suggère que ces processus individuels sont influencés par d'autres agents (pairs et formateurs) qui fournissent des indicateurs d'efficacité personnelle qui teintent l'autoévaluation de l'enseignant. Cette contribution à l'auto-évaluation, combinée à l'enseignement de pratiques novatrices, intensifie le sentiment d'efficacité personnelle, sentiment qui, à son tour, influence les buts fixés et les efforts fournis par l'enseignant. Les changements de buts et l'effort fourni contribuent pour leur part au développement 
de pratiques professionnelles qui conduisent à une amélioration de la réussite scolaire des élèves.

À partir du modèle de Ross et Bruce (2007), la figure 3 présente le processus de changement attendu chez l'enseignant participant au programme de formation à la gestion positive des situations de classe (GPS).

\section{Figure 3 - Processus de changement chez l'enseignant en formation}

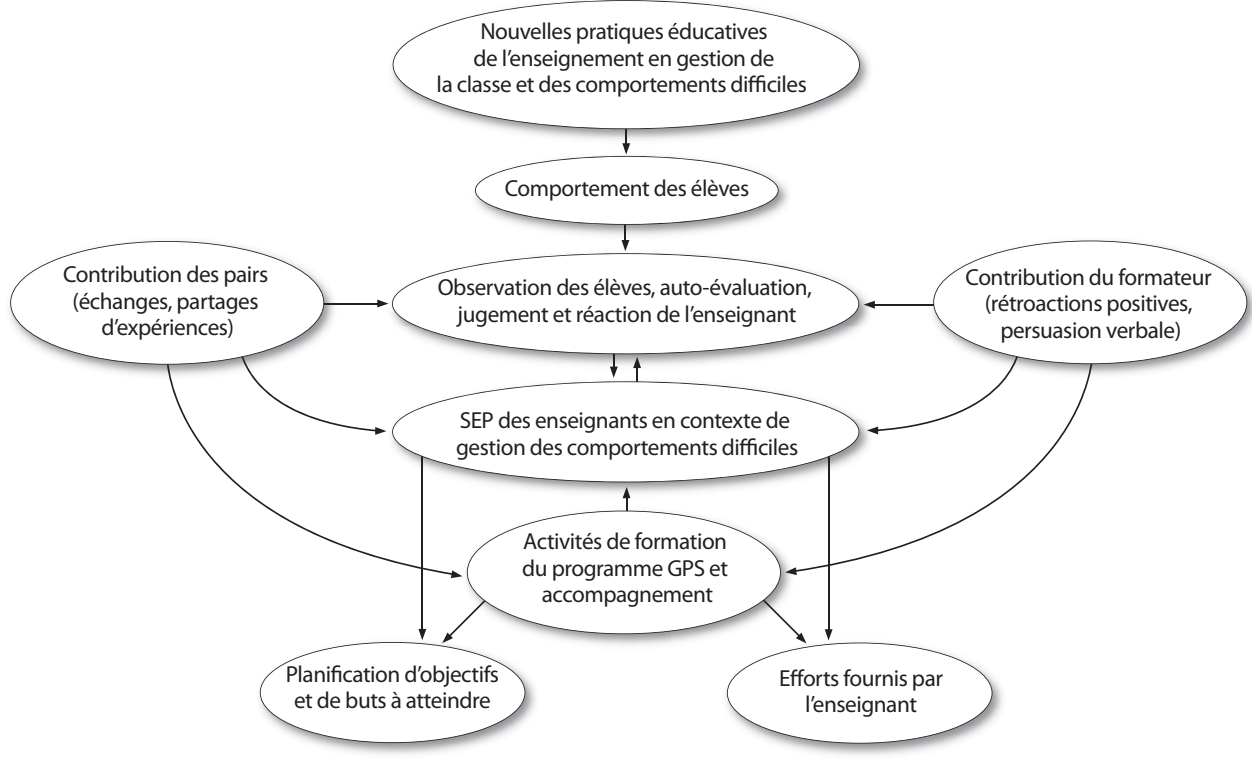

Les modalités d'animation et d'accompagnement représentent des sources potentielles de développement des croyances d'efficacité de l'enseignant. La contribution des pairs et de l'animateur influence le processus d'auto-évaluation de l'enseignant, ses croyances d'efficacité, ainsi que le jugement qu'il porte sur le comportement des élèves et le développement de pratiques pédagogiques renouvelées. Tel que soutenu par la théorie d'auto-efficacité de Bandura (2003), les croyances d'efficacité de l'enseignant influencent le processus d'auto-évaluation et le jugement qu'il porte sur les comportements de ses élèves. En contrepartie, le jugement porté par l'enseignant concernant sa capacité à intervenir efficacement auprès des élèves qui présentent des comportements difficiles influence à son tour les croyances d'efficacité de l'enseignant. Pour leur part, les croyances d'efficacité de l'enseignant et 
le développement de nouvelles approches d'intervention et de nouvelles connaissances influencent la planification des objectifs et des buts à atteindre et les efforts fournis par l'enseignant. Ces derniers agissent sur ses pratiques pédagogiques en classe, facteur déterminant de la réussite des élèves.

Cette contribution à l'auto-évaluation combinée à l'enseignement de pratiques novatrices intensifie le sentiment d'efficacité personnelle qui, à son tour, influence les buts fixés et les efforts fournis par l'enseignant. Les changements de buts et l'effort fourni contribuent pour leur part au développement des pratiques professionnelles qui conduisent à une amélioration de la réussite scolaire des élèves.

\section{Conclusion}

Cet article avait pour but de présenter les prémisses de base ayant mené à l'élaboration du programme de formation GPS. Il constitue un outil de formation continue original conçu à partir de la littérature scientifique qui s'est intéressée aux domaines de la formation continue, des interventions en gestion de classe ainsi qu'à partir de la théorie sociocognitive de l'auto-efficacité de Bandura. Le programme de formation GPS est unique en son genre. D'abord, il innove en permettant d'offrir aux enseignants la possibilité de développer leurs compétences en gestion de la classe et des comportements difficiles, tout en portant une attention particulière aux facteurs d'influence du processus de changement de pratiques éducatives de ces derniers (tel que présenté dans la figure 3). Bien qu'il ait été conçu afin de répondre d'abord aux besoins des enseignants du premier cycle du primaire ( $1^{\text {ière }}$ et $2^{\mathrm{e}}$ années du primaire), il s'avère tout aussi pertinent pour les enseignants des autres cycles de l'école primaire, voire du secondaire. Par la promotion de saines pratiques éducatives auprès des enseignants appelés à encadrer de jeunes élèves qui éprouvent des difficultés d'adaptation à l'école, il constitue un moyen novateur de prévention des problèmes de comportement à l'école. Finalement, il a été conçu de manière à permettre aux enseignants de vivre une expérience optimale de formation en cours d'emploi. Pour atteindre cet objectif, le modèle propose des activités de formation, des modalités d'animation, de soutien et d'accompagnement qui favorisent le changement de pratiques d'intervention en gestion des comportements difficiles en classe, tout en développant de plus fortes croyances d'efficacité personnelle chez les enseignants. Une première étude des effets du programme de formation GPS sur les 
croyances d'efficacité des enseignants a été réalisée auprès d'un groupe d'enseignants du premier cycle de la région de Québec. Les résultats de cette étude s'avèrent positifs et laissent entrevoir des retombées encourageantes sur le plan des pratiques de gestion des comportements difficiles en classe (Gaudreau, 2011a, 2011b).

\section{Références}

Allen, S. J., \& Blackston, A. R. (2003). Training preservice teachers in collaborative problem solving: An investigation of the impact on teacher and student behavior change in real-world settings. School Psychology Quarterly, 18, 22-51.

Alvarez, H. K. (2007). The impact of teacher preparation on responses to student aggression in the classroom. Teaching and Teacher Education, 23, 1113-1126.

Archambault, J. \& Chouinard, R. (2005). Les dix conditions d'une gestion de classe efficace. Vivre le primaire, 18, 30-35.

Archambault, J., \& Chouinard, R. (2009). Vers une gestion éducative de la classe. Montréal, Canada: Gaëtan Morin.

Badia Martin, M. D. M. (2006). Disruptive behaviour in schools. Education Journal, 92, 32-35.

Baker, C. N., Kupersmidt, J. B., Voegler-Lee, M. E., Arnold, D. H., \& Willoughby, M. T. (2010). Predicting teacher participation in a classroombased, integrated preventive intervention for preschoolers. Early Childhood Research Quarterly, 25, 270-283.

Baker, P. H. (2005). Managing student behavior: How ready are the teachers to meet the challenge? American Secondary Education, 33, 51-64.

Bandura, A. (1977). Self-efficacy: Toward a unifying theory of behavioral change. Psychological Review, 84, 191-215.

Bandura, A. (1986). Social foundations of thought and action: A social cognitive theory. Englewood Cliffs, NJ: Prentice-Hall.

Bandura, A. (1991). Self-efficacy mechanism in physiological activation and health promoting behavior. In J. Madden (Ed.), Neurobiology of learning, emotion and affect (pp. 229-270). NewYork, NY: Raven.

Bandura, A. (2003). Auto-efficacité: le sentiment d'efficacité personnelle. Paris: Éditions De Boeck.

Beaman, R., \& Wheldhall, K. (2000). Teachers' use of approval and disapproval in the classroom. Educational Psychology, 20, 431-446.

Begeny, J. C., \& Martens, B.K. (2006). Assessing pre-service teachers' training in empirically-validated behavioral instruction practices. School Psychology Quarterly, 21, 262-285.

Bertsch, K. M., Houlihan, D., Lenz, M. A., \& Patte, C. A. (2009). Teachers commands and their role in preschool classrooms. Electronic Journal of Research in Educational Psychology, 7, 133-162. 
Bissonnette, S., \& Richard, M. (2010). Les modalités d'efficacité de la formation continue. Vivre le primaire, 23, 24-36.

Boyle, B., Lamprianou, I., \& Boyle, T. (2005). A longitudinal study of teacher change: What makes professional development effective? Report of the second year of the study. School Effectiveness and School Improvement, $16,1-27$.

Boynton, M., \& Boynton, C. (2009). Prévenir et régler les problèmes de discipline. Adaptation de Mylène Mercier. Montréal, Canada: Chenelière Éducation.

Brouwers, A., \& Tomic, W. (2000). Disruptive student behavior, perceived selfefficacy, and teacher burnout. Paper presented at the Annual Meeting of the American Psychological Association, Washington, D.C.

Brouwers, A., \& Tomic, W. (2001). The factorial validity of scores on the teacher interpersonal self-efficacy scale. Educational and Psychological Measurement, 61, 433-45.

Bullock, L. M., Ellis, L. L., \& Wilson, M. J. (1994). Knowledge/skills needed by teachers who work with students with severe emotional/behavioural disorders: A revisitation. Behavioral Disorders, 19, 108-125.

Burke, R. J., Greenglass, E. R., \& Shwarzer, R. (1996). Predicting teacher burnout over time: Effects of work stress, social support, and self-doubts on burnout and its consequences. Anxiety, Stress and Coping, 9, 261275.

Buyse, E., Verschueren, K., Doumen, S., Van Damme, J., \& Maes, F. (2008). Classroom problem behavior and teacher-child relationships in kindergarten: The moderating role of classroom climate. Journal of School Psychology, 46, 367-391

Canter, L., \& Canter, M. (2001). Assertive discipline: positive behavior management for today's classroom (3rd ed.). Santa Monica, CA: Lee Canter \& Associates.

Carleton, L. E., Fitch, J.C., \& Krockover, G. H. (2008). An in-service teacher education program's effect on teacher efficacy and attitudes. The Educational Forum, 72, 46-62.

Coalition for Evidence-Based Policy (2002). Bringing evidence-driven progress to education: A recommended strategy for the US Department of Education. Report of the Coalition for Evidence-Based Policy. Retrieved from http://www.eric.ed.gov/PDFS/ED474378.pdf

Conseil des ministres de l'Éducation (2002). Formation du personnel enseignant, des éducatrices et éducateurs: Tendances actuelles et orientations futures. Rapport du Colloque du programme pancanadien de recherche en éducation 2001. Récupéré de http://dsp-psd.pwgsc.gc.ca/ Collection/Statcan/81-593-X/81-593-XIF2001001.pdf 
Conseil supérieur de l'éducation (2001). Les élèves en difficulté de comportement à l'école primaire: comprendre, prévenir, intervenir. Avis au ministre de l'Éducation. Bibliothèque nationale du Québec. Retrieved from http://www.cse.gouv.qc.ca/fichiers/documents/publications/dif_ comp.pdf

Corcoran, T.B. (1995). Helping teachers teach well:Transforming professional development. CPRE Policy Briefs. Consortium for policy research in education, New Brunswick, NJ. Retrieved from http://www.cpre.org/ images/stories/cpre_pdfs/rb16.pdf

Corcoran, T. B., Shields, P. M., \& Zucker, A. A. (1998). Evaluation of NSF's statewide systemic initiatives (SSI) program: The SSIs and professional development for teachers. Menlo Park, CA: RI International. Retrieved from http://policyweb.sri.com/cep/publications/ssiprfdv.pdf

Couture, C. (2005). Les enseignants du primaire face au trouble déficitaire d'attention/hyperactivité. Vie pédagogique, 135, 1-8.

Darling-Hammond, L. (1995). Changing conceptions of teaching and teacher development. Teacher Education Quarterly, 22, 9-26.

Dembo, M.H., \& Gibson, S. (1985). Teachers' sense of efficacy: An important factor in school improvement. The Elementary School Journal, 86, 173184.

Dickens-Smith, M. (1995). The effect of inclusion training on teacher attitude towards inclusion. Chicago, IL: Chicago Public Schools. Retrieved from http://www.eric.ed.gov/PDFS/ED381486.pdf

Doyle, W. (2006). Ecological approches to classroom Management. In C. M. Evertson \& C. S. Weinstein (Eds.), Handbook of classroom management: Research, practice, and contemporary issues (pp. 97-125). Mahwah, NJ: Lawrence Erlbaum Associates.

Ellis, J. (1997). What a seriously at risk student would really like to say to teachers about classroom management. Education Canada, 37, 17.

Evertson, C. (1989). Improving elementary classroom management:A schoolbased training program for beginning the year. Journal of Educational Research, 83, 82-90.

Evertson, C. M., \& Emmer, E. T. (2009). Classroom management for elementary teachers (8th ed.). Upper Saddle River, NJ: Pearson Education, Inc.

Evertson, C. M., \& Weinstein, C. S. (2006). Handbook of classroom management: Research, practice, and contemporary issues. Mahwah, NJ: Lawrence Erlbaum Associates.

Fritz, J. J., Miller-Heyl, J., Kreutzer, J. C., \& MacPhee, D. (1995). Fostering personal teaching efficacy through staff development and classroom activities. Journal of Educational Research, 88, 200-208.

Gaudreau, N. (2011a). Les comportements difficiles en classe: les effets d'une formation continue sur le sentiment d'efficacité des enseignants. (Thèse de doctorat non publiée), Université Laval, Québec. 
Gaudreau, N. (2011b). La formation à la gestion positive des situations de classe (GPS): une bonne manière de prévenir les problèmes de comportement chez les jeunes du primaire. Vie pédagogique, 156. Retrieved from http://www.mels.gouv.qc.ca/sections/viepedagogique/156/ index.asp?page $=$ dossierB_1

Gaudreau, N. (2008). Programme de formation à la gestion positive des situations de classe (GPS). Commission scolaire de la Capitale. Québec, Canada.

Garet, M. S., Porter, A. C., Desimone, L., Birman, B. F., \& Yoon, K.S. (2001). What makes professional development effective? Results from a national sample of teachers. American Educational Research Journal, 38, 915945.

Gersten, R., Keating, T., Yovanoff, P., \& Harniss, M. K. (2001). Working in special education: Factors that enhance special educators intent to stay. Exceptional Children, 67, 549-567.

Gordon, L. M. (2001). High teacher efficacy as marker of teacher effectiveness in the domain of classroom management. Paper presented at the Annual Meeting of the California Council on Teacher Education, San Diego, CA.

Gray, P., Miller, A., \& Noakes, J. (1996). Challenging behaviour. London, UK: Routhledge.

Hamre, B. K., \& Pianta, R. C. (2001). Early teacher-child relationship and the trajectory of children's school outcomes through eighth grade. Child Development, 72, 625-638.

Hamre, B. K., \& Pianta, R. C. (2005). Can instructional and emotional support in the first-grade classroom make a difference for children at risk of school failure? Child Development, 76, 949-967.

Hastings, R. P. (2005). Staff in special education settings and behaviour problems: Towards a framework for research and practice. Educational Psychology, 25, 207-221.

Hastings, R. P., \& Bham, M. S. (2003). The relationship between student behaviour patterns and teacher burnout. School Psychology International, 24, 115-127.

Helm, C. (2007). Teacher dispositions affecting self-esteem and student performance. The Clearing House, 80, 109-110.

Hoffman, L. L., Hutchinson, C. J., \& Reiss, E. (2009). On improving school climate: Reducing reliance on rewards and punishment. International Journal of Whole Schooling, 5, 13-23.

Hustler, D., McNamara, O., Jarvis, J., Londra, M., Campbell, A., \& Howson, J. (2003). Teachers' Perspectives of Continuing Professional Development: DfES Research Report No. 429, London, UK: DfES.

Jeffrey, D., \& Sun, F. (2006). Enseignants dans la violence. Québec: Presses de l’Université Laval, Canada. 
Jolivette, K., \& Steel, E. A. (2010). Classroom management strategies for young children with challenging behavior within early childhood settings. NHSA Dialog, 13, 198-213.

Jones, H. A., \& Chronis-Tuscano, A. (2008). Efficacy of teacher in-service training for attention-deficit/hyperactivity disorder. Psychology in the Schools, 45, 918-929.

Jones, V. F., \& Jones, L. S. (2007). Comprehensive classroom management, creating communities of support and solving problems (8th ed.). Needham Heights, MA: Allyn and Bacon.

Joyce, B., \& Showers, B. (2002). Student achievement through staff development.Alexandria, VA:Association for Supervision and Curriculum Development.

Kariuki, C. (2009). Professional development for 21st century teachers: Effective classroom management. Jackson State University. Récupéré de http://www.eric.ed.gov/PDFS/ED505988.pdf

Kauffman, J. M., Mostert, M. P., Trent, S. C., \& Pullen, P. L. (2006). Managing classroom behaviour: A reflective case-based approach (4thed.). Boston, MA: Allyn \& Bacon / Merrill.

Kehle, T. J., Bray, M. A., Theodore, A. A., Jenson, W. R., \& Clark, E. (2000). A multi-component intervention designed to reduce disruptive classroom behaviour. Psychology in the Schools, 37, 475-481.

Kovess-Masféty, V., Rios-Seidel, C., \& Sevilla-Dedieu, C. (2007). Teachers' mental health and teaching levels. Teaching and Teacher Education, 23, 1177-1192.

Levin, B. B., \& Rock, T. C. (2003). The effects of collaborative action research on preservice and experienced teacher partners in professional development schools. Journal of Teacher Education, 54, 135-149.

Lewis, V.E.(2001).User assessments of glasser-based behavioral management inservice programs for teachers. (Thèse de doctorat non publiée). Drake University, Des Moines, IA.

Lieberman, A. (1996). Creating intentional learning communities. Educational Leadership, 54, 51-55.

Lindauer, P., Petrie, G., Garth, L., \& Vickers, L. (1998). Five keys to successful non-grades primary schools in Hardin County, Kentuky. Reading Improvement, 35, 38-44.

Little, W. (1993). Teachers' professional development in a climate of educational reform. Educational Evaluation and Policy Analysis, 15, 129-151.

Massé, L., Desbiens, N., \& Lanaris, C. (2006). Les troubles du comportement à l'école. Prévention, évaluation et intervention. Montréal, QC: Gaëtan Morin.

Matheson, A. S., \& Shriver, M. D. (2005). Training teachers to give effective commands: Effects on student compliance and academic behaviours. School Psychology Review, 34, 202-219. 
Mayer, G. R. (2001). Antisocial behavior: Its causes and prevention within our schools. Education and Treatment of Children, 24, 414-429.

McEvoy, J., McDonnell, A., \& Dearden, B. (1991). Challenging behaviour in the classroom. British Journal of Special Education, 18, 141-143.

Melby, L. C. (1995). Teacher efficacy and classroom management: A study of teacher cognition, emotion and strategy usage associated with externalizing student behaviour (Thèse de doctorat non publiée). Université de Californie, Oakland, CA.

Merrett, F., \& Wheldall, K. (1993). How do teachers learn to manage classroom behaviour? A study of teachers' opinions about their initial training with special reference to classroom behaviour management, Educational Studies, 19, 91-105.

Milne, D. (1986). Training nurses as behaviour therapists. Londres, U.K.: Croom Helm.

Ministère de l'éducation du Québec (MEQ) (2001). La formation à l'enseignement: Les orientations, les compétences professionnelles. Québec, Canada: Gouvernement du Québec.

MELS. (in press). Cadre de référence visant à soutenir les milieux concernant les modalités d'intervention à l'intention des élèves ayant des difficultés de comportement. Direction de l'adaptation scolaire et des services complémentaires. Québec, Canada : Gouvernement du Québec.

Murik, J., Shaddock, A., Spinks, A., Zilber, D., \& Curry, C. (2005). Reported strategies for responding to the aggressive and extremely disruptive behaviour of students who have special needs. Australasian Journal of Special Education, 29, 21-39.

Myers, S. S., \& Pianta, R. C. (2008). Developmental commentary: Individual and contextual influences on student-teacher relationship and children's early problem behaviors. Journal of Clinical Child and Adolescent Psychology, 37, 600-608.

Ndoreraho, J.-P., \& Martineau, S. (2006). Une problématique des débuts de la carrière en enseignement, Retrieved from http://www.insertion.qc.ca/ spip.php?article104

Ohlhausen, M., Meyerson, M., \& Sexton, T. (1992). Viewing innovations through the efficacy-based change model: A whole language application. Journal of Reading, 35, 536-541.

Parent, G., Cartier, R., Laurin, P., Lavoie, L., Rhéaume, D., Toussaint, P., Jean, C. (1997). Les perceptions d'enseignantes et d'enseignants en adaptation scolaire quant à la formation continue. Scientia Paedagogica Experimentalis, 24, 117-154.

Patterson, G. R., Reid, J. B., \& Dishion, T. J. (1992). A social learning approach: IV. Antisocial boys. Eugene, OR: Castalia Publishing Co.

Perkins, J., \& Leadbetter, D. (2002). An evaluation of aggression management training in a special education setting. Emotional and Behavioural Difficulties, 7, 19-34. 
Poulou, M. (2005). Perceptions of students with emotional and behavioural difficulties. Emotional and behavioural difficulties, 10, 137-160.

Poulou, M. (2007). Personal teaching efficacy and its sources: student teachers' perceptions. Educational Psychology, 27, 191-218.

Rimm-Kaufman, S. E., \& Sawyer, B. E. (2004). Primary-grade teachers' selfefficacy beliefs, attitudes toward teaching, and discipline and teaching practice priorities in relation to the responsive classroom approach. The Elementary School Journal, 104, 321-341.

Robardey, C. P., Allard, D. W., \& Brown, D. M. (1994). An assessment of the effectiveness of full option science system training for third- through sixth-grade teachers. Elementary Science Education, 6, 17-29.

Rosenberg, M. S., Sindelar, P. T., \& Hardman, M. L. (2004). Preparing highly qualified teachers for students with emotional or behavioral disorders: The impact of NCLB and IDEA. Behavioral Disorders, 29, 266-278.

Ross, J.A. (1994). The impact of an in service to promote cooperative learning on the stability of teacher efficacy. Teaching \& Teacher Education, 10, 381-394.

Ross, J. A. \& Bruce, C. (2007). Professional development effects on teacher efficacy: Results of randomized field trial. The Journal of Educational Research, 101, 50-60.

Royer, É. (2006). Le chuchotement de Galilée. Québec, Canada: Corporation École et Comportement.

Sawka, K. D., McCurdy, B. L., \& Mannella, M. C. (2002). Strengthening emotional support services: An empirically based model for training teachers of students with behaviour disorders. Journal of Emotional and Behavioural Disorders, 10, 223-232.

Shernoff, E. S., \& Kratochwill, T. R. (2007). Transporting an evidence-based classroom management behavior problem to a school: An analysis of implementation, outcomes, and contextual variables. School Psychology Quarterly, 22, 449-472.

Shields, P. M., Marsh, J. A., \& Adelman, N. E. (1998). Evaluation of NSF.s Statewide Systemic Initiatives Program: The SSI.s impact on classroom practice. Menlo Park, CA: SRI.

Siegel, J., \& Jausovec, N. (1994). Improving teachers' attitudes toward students with disabilities. Paper presented at the International Council on Education for Teaching Conference. Istanbul, Turkey.

Skaalvik, E. M., \& Skaalvik, S. (2007). Dimensions of teacher self-efficacy and relations with strain factors, perceived collective teacher efficacy, and teacher burnout. Journal of Educational Psychology, 99, 611-625.

Skiba, R. J., \& Peterson, R. L. (2000). School discipline at a crossroads: From zero tolerance to early response. The Council for Exceptional Children, $66,335-347$. 
Stein, M., \& Wang, M. (1988). Teacher development and school improvement: The process of teacher change. Teaching and teacher Education, 4, 171187.

Swinson, J., \& Cording, M. (2002). Assertive discipline in a school for pupils with emotional and behavioural difficulties. British Journal of Special Education, 29, 72-75.

Veenman, S., Lem, P., \& Roelofs, E. (1989). Training teachers in mixedage classrooms: Effects of staff development programme. Educational Studies, 15, 165-180.

Walker, H. M., Ramsey, E., \& Gresham, F. M. (2004). Antisocial behavior in school: Strategies and best practices (2nd edition). Pacific Grove, CA: Brooks/Cole.

Weiss, I. R., Montgomery, D. L., Ridgway, C. J., \& Bond, S. L. (1998). Local systemic change through teacher enhancement: Year three cross-site report. Chapel Hill, NC: Horizon Research.

Wheldall, K., \& Merrett, F. (1992). Effective classroom behaviour management: Positive teaching. In K. Wheldall (Ed.), Discipline in schools: Psychological perspectives on the Elton report (pp. 46-65). New York, NY: Routledge.

Wisniewski, L., \& Gargiulo, R. M. (1997). Occupational stress and burnout among special educators: A review of the literature. Journal of Special Education, 31, 325-346.

Woolfolk, A. (2004). Educational Psychology (9th ed.), Boston, MA: Allyn \& Bacon.

\section{Notes}

1 Organisme américain à but non lucratif visant à accroître l'efficacité du gouvernement par l'utilisation des résultats de recherche probants (domaine médical, social et de l'éducation).

2 Le MELS (Ministère de l'Éducation, du Loisir et du Sport, province du Québec) publiait en 2001 son cadre de référence sur la formation à l'enseignement qui présente les orientations et le référentiel des douze compétences professionnelles à développer chez le personnel enseignant. Chacune de ces compétences s'opérationnalise à l'aide de composantes prenant la forme de comportements attendus chez l'enseignant. 


\section{Correspondance}

Nancy Gaudreau

Département des sciences de l'éducation

Université du Québec à Trois-Rivières

C.P. 500

Trois-Rivières, Québec, Canada G9A 5H7

Téléphone : (819) 376-5011, poste 3617

Courriel : nancy.gaudreau@uqtr.ca

\section{Résumé}

Plusieurs auteurs dénoncent depuis quelques années le manque de formation des enseignants pour gérer efficacement les comportements difficiles des élèves. Afin de soutenir la réussite des élèves qui présentent des difficultés de comportement, il s'avère essentiel de développer des modèles de formation en cours d'emploi qui répondent aux besoins des enseignants. Cet article présente le programme de formation à la gestion positive des situations de classe (GPS), spécialement conçu pour les enseignants du premier cycle du primaire à partir des données probantes de recherches dans le domaine. Il vise le développement des compétences professionnelles des enseignants en matière de gestion de la classe et des comportements difficiles. Les formules pédagogiques proposées ont pour but de soutenir le développement des croyances d'efficacité personnelle chez les enseignants afin de favoriser l'implantation de pratiques éducatives permettant de prévenir les comportements difficiles et de les gérer efficacement en classe.

Mots-clés : sentiment d'efficacité personnelle; formation continue; difficultés de comportement; programme de formation; enseignant; primaire.

$$
* * *
$$

\section{Abstract}

Several authors question that the preparation of teachers to effectively manage challenging behaviors in the classroom is insufficient. To support the success of students with behavioral difficulties, it is essential to develop models of training that meet the needs of teachers. This article presents the inservice training program of classroom management, the 
Programme de formation à la gestion positive des situations de classe (GPS), specially designed for first- and second-grade teachers. It aims to develop professional skills of teachers in classroom management and challenging behaviors. The teaching formulas are proposed to support the development of self-efficacy beliefs among teachers to promote the implementation of educational practices to prevent and deal effectively with difficult behaviors in the classroom.

Keywords : teacher self-efficacy; inservice training; behavioral disorder; training program; teacher; elementary. 